\title{
Diagnóstico situacional das Práticas Integrativas e Complementares na Atenção Primária à Saúde do Maranhão
}

\author{
Situational diagnosis of Integrative and Complementary Practices in \\ Primary Health Care of Maranhão
}

Rafaela Duailibe Soares ${ }^{\mathbf{1}}$, Judith Rafaelle Oliveira Pinho', Aline Sampieri Tonello'

DOI: $10.1590 / 0103-1104202012612$

\begin{abstract}
RESUMO Esta pesquisa apresenta o diagnóstico situacional das Práticas Integrativas e Complementares na Atenção Primária à Saúde do estado do Maranhão. Foi realizado um estudo descritivo, com dados primários dos 217 municípios do estado, através de um formulário enviado a todos os secretários municipais de saúde, no período de abril a julho de 2019. A taxa de resposta foi de $100 \%$, indicando que $25,4 \%$ dos municípios ofertam Práticas Integrativas e Complementares. Fitoterapia e massoterapia são as mais ofertadas, estando presentes em 49,1\% e 29,1\% dos municípios, respectivamente. Os fisioterapeutas são os profissionais que mais executam as Práticas Integrativas e Complementares (54,5\% dos municípios). Em 49,1\% dos municípios, essas práticas são ofertadas pela Estratégia Saúde da Família, e em 47,3\%, pelo Núcleo Ampliado de Saúde da Família. No que diz respeito aos instrumentos de gestão, organização e legislação específica, observou-se que nenhum município do estado possui legislação específica para as práticas e $14,5 \%$ dos municípios preveem alguma estrutura organizacional específica para geri-las. A oferta destas práticas no Maranhão ainda é um desafio, mesmo com as orientações da Organização Mundial de Saúde e a instituição da Política Nacional de Práticas Integrativas e Complementares no Sistema Único de Saúde.
\end{abstract}

PALAVRAS-CHAVE Terapias complementares. Atenção Primária à Saúde. Diagnóstico da situação de saúde.

\begin{abstract}
This research presents the situational diagnosis of the Integrative and Complementary Practices in Primary Health Care of Maranhão state. A descriptive study, with primary data from the 217 municipalities of the state was carried out, through a form sent to all municipal health secretaries, from April to July 2019. The response rate was $100 \%$, identifying that $25.4 \%$ of the municipalities offer Integrative and Complementary Practices. Phytotherapy and massage therapy are the most offered, being present in $49.1 \%$ and $29.1 \%$ of the municipalities, respectively. Physical therapists are the professionals who most perform Integrative and Complementary Practices (54.5\% of the municipalities). In 49.1\% of the municipalities, these practices are offered by the Family Health Strategy, and in 47.3\%, by the Extended Family Health Center. Regarding the management, organization and specific legislation tools, it was observed that no municipality in the state has specific legislation for practices and $14.5 \%$ of the municipalities foresees some specific organizational structure for managing them. The offer of these practices in Maranhão is still a challenge, even with the guidelines of the World Health Organization and the establishment of the National Policy of Integrative and Complementary Practices in the Unified Health System.
\end{abstract}

1 Universidade Federal do Maranhão (UFMA) - São Luís (MA), Brasil. rafaela.duailibe@gmail. com
KEYWORDS Complementary therapies. Primary Health Care. Health situation diagnosis. 


\section{Introdução}

A implantação da Medicina Tradicional Chinesa ou Medicina Complementar e Alternativa (MTC/MCA) começou a ser estimulada pela Organização Mundial da Saúde (OMS) na década de 1970, com orientações referentes à estruturação da política, garantia de segurança, qualidade e eficácia, ampliação do acesso e ao uso racional'.

Além dessa estimulação pela OMS para que os países elaborem políticas que incluam as Práticas Integrativas e Complementares em Saúde (Pics) nos serviços públicos, uma discussão mundial sobre uma nova concepção de saúde, bem como o declínio do modelo biomédico, têm valorizado a expansão destas práticas em nível mundial. Parte do crescimento das Pics deve-se aos benefícios que estas práticas geram, bem como à valorização do indivíduo como centro do cuidado, à busca por meios terapêuticos simples, menos dependentes de tecnologia científica dura, mais econômicos e com igual ou maior eficácia nas situações comuns de adoecimento, e à busca pela autonomia do usuário, com foco central na saúde e não na doença ${ }^{2}$.

No Brasil, desde a instituição do Sistema Único de Saúde (SUS), o uso das Pics vem sendo discutido, uma vez que, devido aos processos de descentralização e à participação popular, estas práticas passam a ser entendidas como fundamentais na garantia do direito à saúde. Porém, somente em 2006, foi instituída uma política pública direcionada a essas práticas: Política Nacional de Práticas Integrativas e Complementares (PNPIC), no SUS ${ }^{3}$.

Essa política foi criada através da Portaria $n^{\circ}$ 971, de 03 de maio de 2006, pelo Ministério da Saúde (MS), ampliando o escopo de ações e serviços ofertados pelo SUS e respondendo a uma demanda de inclusão de alguns serviços nesse sistema. A PNPIC incluiu e aprovou no SUS, a acupuntura, a fitoterapia, a homeopatia, o termalismo/crenoterapia, a medicina antroposófica e a MTC. Com isto, é essencial consolidar o entendimento de que estas formas de cuidado devem ser garantidas ao usuário, assim também garantindo o princípio da integralidade do cuidado 4 .

Então, a PNPIC visa normatizar a utilização dessas práticas no SUS, não apenas convergindo com os princípios de integralidade e universalidade, mas também contribuindo para o aumento da resolubilidade do sistema ${ }^{5}$.

No ano de 2004, foi realizada uma pesquisa nacional sobre as práticas e as Pics mais frequentemente realizadas nos municípios brasileiros. Foram encontradas: a homeopatia, a MTC/acupuntura, a fitoterapia e a medicina antroposófica. No que tange à presença de lei ou ato municipal normatizador de ações e serviços, apenas $6 \%$ dos estados/municípios apresentavam legislação pertinente. Identificou-se, ainda, que as ações de todas as Pics eram constatadas, preponderantemente, na atenção básica e na Estratégia Saúde da Família (ESF) ${ }^{6}$.

A partir da Portaria no 849 , de 27 de março de 2017, o MS ampliou o escopo de Pics ofertadas pelo SUS com o objetivo de atender às diretrizes da OMS e avançar na institucionalização dessas práticas no âmbito do sistema, passando a incluir arteterapia, ayurveda, biodança, dança circular, meditação, musicoterapia, naturopatia, osteopatia, quiropraxia, reflexoterapia, reiki, shantala, terapia comunitária integrativa e yoga ${ }^{7}$.

Em 21 de março de 2018, dez novas Pics foram incluídas na PNPIC, de acordo com a Portaria $n^{0} 702$ : apiterapia, aromoterapia, bioenergética, constelação familiar, cromoterapia, geoterapia, hipnoterapia, imposição das mãos, ozonioterapia e terapias de florais ${ }^{7}$.

Entre os benefícios alcançados pelo uso das Pics no cuidado à saúde, destacam-se: relaxamento, bem-estar, alívio de dor e de ansiedade, redução do uso de medicamentos, prevenção de doenças, fortalecimento do sistema imunológico e diminuição de reações adversas em comparação com o uso exclusivo de tratamento medicamentoso 22 .

Deve-se ressaltar que o uso das Pics no SUS como política nacional ainda é um desafio, uma 
vez que, no Brasil, ainda há supervalorização e incentivo à aplicação de insumos tecnológicos sofisticados e de alto dispêndio, predomínio do modelo biomédico, falta de conhecimento, carência de capacitação de recursos humanos no próprio SUS, na área, e baixa oferta de disciplinas na formação acadêmica ${ }^{23}$. Não obstante, as Pics se apresentam com potencial para favorecer a desmedicalização nos serviços de saúde ${ }^{25}$.

O desenvolvimento das Pics pode estar relacionado ao resgate de valores perdidos na dimensão terapêutica da biomedicina. Tais práticas terapêuticas suprem uma demanda social, já que oferecem soluções alternativas para o sofrimento, sendo este o principal motivo de seu incentivo, ainda que apenas pela sociedade civil26.

O esforço de implementação e expansão dessas práticas geralmente parte dos profissionais de saúde com engajamento nas Pics, estimulados pelo objetivo de consolidarem uma concepção de cuidado divergente do modelo preponderante, entendendo que há práticas alternativas que podem se destacar quanto aos resultados, tornando-se parte de um novo processo de implementação de maneiras alternativas de promoção da saúde, que compreendem o ser humano em sua totalidade, ${ }^{\mathbf{8}, 27}$.

Considera-se, ainda, que o novo Regime Fiscal, instituído pela Emenda Constitucional $\mathrm{n}^{\circ} 95$ (EC 95/2016) - que congela o gasto primário federal por 20 anos, com profundos impactos no financiamento da saúde - tornará mais difícil a efetivação do direito à saúde, disponibilizando para o mercado de planos de saúde aqueles que dispõem de recursos financeiros; para a judicialização, os que conhecem as responsabilidades do estado, bem como seus direitos e meios de exigi-los; e insuficiência da oferta e da qualidade dos serviços públicos para os mais vulneráveis9.

Diante desse contexto, considera-se imperiosa a necessidade de identificar os municípios que realizam serviços de Pics, em especial, no estado do Maranhão, já que não se observaram estudos capazes de informar a situação das Pics no estado. Acredita-se ser oportuna a realização do estudo proposto, principalmente quanto à ampliação de conhecimentos, reflexão e incorporação de sugestões para o fortalecimento das referidas práticas no SUS, no estado, uma vez que há carência de informações sobre a oferta de serviços relacionados a tais práticas. O presente artigo teve como objetivo realizar o diagnóstico situacional das Pics na Atenção Primária à Saúde (APS) do estado do Maranhão.

\section{Material e métodos}

Trata-se de um estudo descritivo, cujas unidades de análise foram os municípios do Maranhão, um dos 27 estados do Brasil, que ocupa uma área de $329.642,182 \mathrm{~km}^{2}$, dividido em 217 municípios. Possui população estimada em 7.075.181 habitantes e apresenta densidade de 19,81 hab $/ \mathrm{km}^{2} \mathbf{2 8}$.

Os 217 municípios do estado estão organizados em 19 regiões de saúde e 3 macrorregiões: Macro Norte, Macro Leste e Macro Sul, que são espaços territoriais homologados na Comissão Intergestores Bipartite, por meio da Resolução $\mathrm{n}^{0}$ 64/2018 - CIB/MA29.

A coleta de dados foi realizada de abril a julho de 2019, através de um questionário eletrônico. Foi realizado um estudo piloto para a aplicação do instrumento de coleta, com oito indivíduos para ajustes necessários.

Antes do envio do questionário, foi realizada uma apresentação na Secretaria de Estado da Saúde do Maranhão para os 18 gestores regionais e para a Coordenação Estadual das Regionais. Além disso, a pesquisa e o instrumento foram apresentados para o presidente do Conselho de Secretários Municipais de Saúde do Maranhão (Cosems), que consentiu e autorizou a realização da pesquisa. O Cosems é uma entidade civil de direito privado, sem fins lucrativos, com autonomia administrativa, financeira e patrimonial. O órgão reúne os secretários municipais de saúde do estado e atua com o objetivo de integrá-los entre si, de forma técnica e administrativa, a fim de favorecer 
a coletividade na aplicação dos serviços de saúde, além de lutar pela hegemonia dos municípios, funcionando como órgão permanente para a troca de experiências.

As variáveis de análise coletadas na pesquisa proposta foram: existência do cargo de coordenador da APS; formação do coordenador da APS; formação do responsável pelo preenchimento do instrumento; existência de ações referentes às Pics; existência de responsável pela coordenação das ações; formação do coordenador dessas ações; oferta de Pics; profissionais que realizam as Pics; normativas para a criação do serviço de Pics; e se as Pics são planejadas nos instrumentos de gestão dos municípios.

Os dados coletados foram tratados com o auxílio do software Microsoft Excel ${ }^{\circledR}$ e exportados para o Stata ${ }^{\circledR} 14.0$ para realização das análises de frequência. Utilizou-se, também, o software Tabwin ${ }^{\circledR}$ para construção do mapa utilizado na demonstração dos resultados.

O desenvolvimento do presente estudo seguiu os requisitos da Resolução ${ }^{0}$ 466, de
12 de dezembro de 2012, do Conselho Nacional de Saúde/Ministério da Saúde, e suas complementares, que regulamentam as pesquisas envolvendo seres humanos. O estudo foi aprovado pelo Comitê de Ética em Pesquisa da Universidade Federal do Maranhão, sob parecer: 3.051.899.

\section{Resultados}

A taxa de resposta do questionário foi de $100 \%$ (217 municípios). Entre os profissionais responsáveis pelo preenchimento do instrumento, 66,4\% (144) foram coordenadores da APS; 17\% (37), secretários de saúde; 8,8\% (19), assessores da APS; 4,1\% (9), coordenadores da vigilância em saúde; e 3,7\% (8), profissionais que realizavam a assistência.

A figura 1 representa espacialmente a distribuição da realização das Pics no Maranhão: 55 municípios ofertam alguma Pics, representando $25,4 \%$ do total de municípios.

Figura 1. Municípios que realizam Pics no Maranhão, 2019

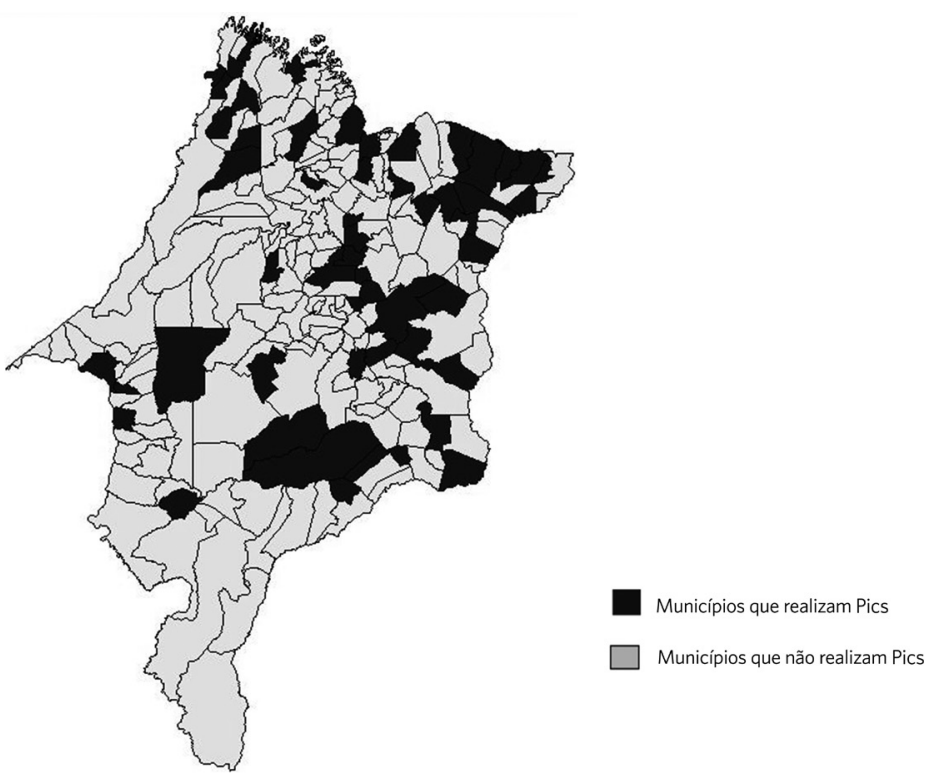


Observa-se que 93,7\% dos municípios apresentam cargo de coordenação da APS e, destes, 90,4\% são enfermeiros. Apenas 10,9\% dos municípios que realizam Pics possuem o cargo 'Coordenação das Pics', dos quais 50\% são ocupados por enfermeiros (tabela 1).

Tabela 1. Características da Coordenação da Atenção Primária em Saúde e da Coordenação das Práticas Integrativas e Complementares em Saúde, Maranhão, 2019

\begin{tabular}{|c|c|c|}
\hline Características da Coordenação da APS e Pics & $\mathbf{N}$ & $\%$ \\
\hline \multicolumn{3}{|l|}{ Existência do cargo de Coordenação de APS } \\
\hline Sim & 208 & 95,8 \\
\hline Não & 9 & 4,2 \\
\hline Total & 217 & 100 \\
\hline \multicolumn{3}{|l|}{ Formação do coordenador da APS } \\
\hline Enfermeiro & 188 & 90,4 \\
\hline Fisioterapeuta & 4 & 1,9 \\
\hline Administrador & 4 & 1,9 \\
\hline Farmacêutico & 3 & 1,4 \\
\hline Outros & 9 & 4,4 \\
\hline Total & 208 & 100 \\
\hline \multicolumn{3}{|l|}{ Existência do cargo de Coordenação das Pics } \\
\hline Sim & 6 & 10,9 \\
\hline Não & 49 & 89,1 \\
\hline Total & 55 & 100 \\
\hline \multicolumn{3}{|l|}{ Formação do coordenador das Pics } \\
\hline Enfermeiro & 3 & 50 \\
\hline Fisioterapeuta & 2 & 33,3 \\
\hline Farmacêutico & 1 & 16,7 \\
\hline Total & 6 & 100 \\
\hline
\end{tabular}

Fonte: Elaboração própria.

Percebe-se que a fitoterapia e a massoterapia figuram entre as Pics mais ofertadas no estado, presentes em 49,1\% e 29,1\% dos municípios que ofertam Pics, respectivamente. Ressalta-se, ainda, que $38,6 \%$ dos municípios que realizam Pics ofertam mais de uma prática (tabela 2).

Os fisioterapeutas são os profissionais que mais executam as Pics no estado, atuando em
54,5\% dos municípios, seguidos dos enfermeiros, com $18,2 \%$ dos municípios. Em 49,1\% dos municípios, estas práticas são ofertadas pela ESF, e em 47,3\%, pelo Núcleo Ampliado de Saúde da Família (Nasf). Em relação ao ano em que as Pics foram iniciadas, percebe-se que 80\% dos municípios passaram a ofertá-las a partir de 2017 (tabela 2). 
Tabela 2. Características da oferta de Práticas Integrativas e Complementares em Saúde, Maranhão, 2019

\begin{tabular}{|c|c|c|}
\hline Características da oferta de Pics & $\mathbf{N}$ & $\%$ \\
\hline \multicolumn{3}{|l|}{ Tipos de Pics ofertadas } \\
\hline Fitoterapia & 27 & 49,1 \\
\hline Massoterapia & 16 & 29,1 \\
\hline Auriculoterapia & 10 & 18,2 \\
\hline Acupuntura & 9 & 16,4 \\
\hline Shantala & 8 & 14,5 \\
\hline Outros & 36 & 65,5 \\
\hline \multicolumn{3}{|c|}{ Frequência do número de Pics ofertadas } \\
\hline 1 Pics & 33 & 60 \\
\hline Mais de 1 Pics & 22 & 40 \\
\hline Total & 55 & 100 \\
\hline \multicolumn{3}{|l|}{ Profissional que realiza as Pics } \\
\hline Fisioterapeuta & 30 & 54,5 \\
\hline Enfermeiro & 10 & 18,2 \\
\hline Outros & 19 & 34,5 \\
\hline \multicolumn{3}{|l|}{ Programa que realiza as Pics } \\
\hline ESF & 27 & 49,1 \\
\hline Nasf & 26 & 47,3 \\
\hline Outros & 2 & 3,6 \\
\hline \multicolumn{3}{|l|}{ Ano que iniciou a oferta das Pics } \\
\hline 2009-2012 & 5 & 9,1 \\
\hline $2013-2016$ & 6 & 10,9 \\
\hline $2017-2019$ & 44 & 80 \\
\hline Total & 55 & 100 \\
\hline
\end{tabular}

Fonte: Elaboração própria.

No que diz respeito aos instrumentos de gestão, organização e legislação específica para as Pics, observa-se que nenhum município do estado possui legislação específica para as práticas, e $14,5 \%$ dos municípios preveem alguma estrutura organizacional específica de gestão das Pics (tabela 3).

Com relação ao Plano Plurianual de Saúde (PPA) e à Programação Anual de Saúde (PAS), $34,5 \%$ e $38,2 \%$ dos municípios afirmam que as Pics estão previstas nestes instrumentos, respectivamente (tabela 3 ). 
Tabela 3. Características da gestão das Práticas Integrativas e Complementares em Saúde, Maranhão, 2019

\begin{tabular}{lrr}
\hline Gestão das Pics & $\mathbf{N}$ & \% \\
\hline Lei Específica & 0 & 0 \\
\hline Sim & 55 & 100 \\
Não & $\mathbf{2 1 7}$ & $\mathbf{1 0 0}$ \\
\hline Pics no Organograma & 8 & 14,5 \\
\hline Sim & 38 & 69,1 \\
Não & 9 & 16,4 \\
Não sei responder & $\mathbf{4}$ & $\mathbf{1 , 9}$ \\
\hline PPA & 19 & 34,5 \\
\hline Sim & 24 & 43,6 \\
Não & 12 & 21,8 \\
Não sei responder & 21 & 38,2 \\
\hline PAS & 22 & 40 \\
\hline Sim & 12 & 21,8 \\
Não & 44 & 100 \\
Não sei responder & 21 \\
Total & 21 \\
\hline
\end{tabular}

Fonte: Elaboração própria.

De acordo com os municípios que ofertam Pics, por região de saúde (tabela 4), destaca-se a região de Codó, com $50 \%$ dos municípios ofertando práticas, e a região de Açailândia, cujos municípios não realizam nenhuma prática. Ressaltam-se, ainda, as regiões de Chapadinha, Rosário, São João dos Patos, Zé Doca e São Luís, com mais de $40 \%$ dos municípios ofertando Pics.

Tabela 4. Número de municípios que ofertam Práticas Integrativas e Complementares em Saúde, por região de saúde, Maranhão, 2019

\begin{tabular}{lrrr}
\hline Região de saúde & № de municípios & № de municípios que ofertam Pics & \% \\
\hline Açailândia & 8 & 0 & 0 \\
Bacabal & 11 & 2 & 18,2 \\
Balsas & 14 & 1 & 7,1 \\
Barra do Corda & 6 & 2 & 33,3 \\
Caxias & 7 & 2 & 28,6 \\
Chapadinha & 13 & 6 & 46,2 \\
Codó & 6 & 3 & 50 \\
Imperatriz & 15 & 4 & 26,7 \\
Itapecuru & 14 & 4 & 28,6 \\
Pedreiras & 13 & 1 & 7,7 \\
Pinheiro & 17 & 3 & 17,7 \\
\hline
\end{tabular}


Tabela 4. (cont.)

\begin{tabular}{lrrr}
\hline Região de saúde & № de municípios & № de municípios que ofertam Pics & $\%$ \\
\hline Presidente Dutra & 16 & 2 & 12,5 \\
Rosário & 12 & 5 & 41,7 \\
Santa Inês & 13 & 1 & 7,7 \\
São João dos Patos & 15 & 6 & 40 \\
Timon & 4 & 1 & 25 \\
Viana & 11 & 2 & 18,2 \\
Zé Doca & 17 & 8 & 47,1 \\
São Luís & 5 & 2 & 40 \\
Total & 217 & 55 & 25,4 \\
\hline
\end{tabular}

Fonte: Elaboração própria.

\section{Discussão}

O presente estudo detectou que, no Maranhão, apenas $25,4 \%$ dos municípios ofertam Pics. Este é um dado primordial para o avanço da PNPIC no estado, uma vez que permite que os gestores conheçam a atual situação da implantação das Pics e sejam sensibilizados e preparados para liderarem e influenciarem a operacionalização destas práticas. Além disto, é necessário o envolvimento de atores sociais, instituições e profissionais, através da gestão participativa, contribuindo para a efetiva implantação das Pics na APS10.

A oferta das Pics no Brasil, especialmente no Maranhão, ainda é um desafio, mesmo com as orientações da OMS, desde a década de 1970, para a implantação de políticas a elas referentes, e mesmo com mais de uma década da instituição da PNPIC no SUS. Isto pode estar relacionado ao fato de a política não prever investimento financeiro adicional para as Pics pela União, sendo uma das poucas políticas nacionais da área da saúde aprovadas sem orçamento próprio ou indutivo, para além do disponível antes de 2006"1.

Além disso, destacam-se alguns fatores que podem estar associados à baixa oferta das práticas no Maranhão, como a falta de coordenação nacional oficial da PNPIC no âmbito do MS, a falta de informação acerca da incorporação de novas Pics, em 2017 e 2018, pelo MS, e a dificuldade de entendimento sobre a aquisição de recursos financeiros para a execução das práticas pelos gestores ${ }^{24}$.

Sua institucionalização no SUS é considerada um desafio para os gestores públicos, também, pela insuficiência de profissionais capacitados para a realização das práticas, pela ausência de financiamento para a política, pelos poucos espaços institucionais para o desenvolvimento de novos serviços e pelas limitações relacionadas aos fatores econômi$\cos$, científicos e organizacionais ${ }^{12}$.

Muitos profissionais de saúde desconhecem a PNPIC, no SUS, o que sugere que esta falta de conhecimento também aconteça no Maranhão, razão pela qual grande parte dos municípios não oferta as Pics na APS'.

Ressalta-se que, devido ao fato de as Pics estarem relacionadas a uma visão holística de saúde, caracterizada por tecnologias leves de cuidado, que ampliam o acesso e garantem a integralidade da atenção à saúde, as relações de vínculo e cuidado integral são importantes para a consolidação do SUS, sobretudo na APS ${ }^{13}$.

Em relação ao perfil dos profissionais que coordenam a APS, na qual as Pics são estratégias fundamentais para a garantia da resolutividade dos problemas, observa-se, 
no presente estudo, a categoria de enfermagem como profissão que ocupa a maioria dos cargos na gestão do estado (90,4\%). Isto pode estar relacionado ao fato de que, entre o rol de atividades desenvolvidas pelo enfermeiro, destacam-se as administrativo-burocráticas, que os afastam gradativamente das atividades assistenciais e os aproximam das atividades gerenciais e administrativas, com ênfase na organização do serviço, no planejamento e no controle do trabalho da equipe ${ }^{14}$. Deste modo, sugere-se especial atenção a esta categoria profissional, no que diz respeito ao fomento da implantação das Pics no estado.

O profissional que ocupa cargos de gestão no SUS deve ter conhecimentos, habilidades e atitudes que possibilitem a execução do seu trabalho com eficiência. Este papel gerencial envolve competências, como o relacionamento interpessoal, a liderança, a motivação da equipe e a comunicação, entre outras, para que ele possa gerenciar as políticas públicas, bem como efetivá-las dentro da APS, como é o caso da PNPIC ${ }^{15}$.

No que diz respeito às coordenações específicas para as Pics, somente $10,9 \%$ dos municípios as possuem. A inexistência de um responsável e/ou coordenador destes serviços pode representar a falta de planejamento dos serviços municipais para a realização de tais práticas, o que dificulta sua consolidação no SUS. Além disto, destaca-se a necessidade de a gestão municipal incorporar a PNPIC aos organogramas das secretarias, principalmente com o objetivo de garantir que ela se efetive.

Na perspectiva da consolidação das Pics nos municípios, estudos afirmam que os profissionais enfrentam dificuldades para desenvolvê-las, na implementação ou na ampliação das ações, embora tenham interesse em fazê-lo. Os obstáculos que se destacam estão, majoritariamente, no campo da gestão ${ }^{\mathbf{1 2}}$.

O resultado do presente estudo, em relação aos tipos de Pics ofertadas, vai ao encontro do afirmado por Tesser, Sousa e Nascimento $(2018)^{\mathbf{1 2}}$, que mencionaram que as Pics ofertadas com maior frequência no Brasil envolvem plantas medicinais e fitoterapia (30\% dos municípios que ofertam Pics), acupuntura (16\%) e auriculoterapia (11\%), distintamente, nas diversas regiões do País. No Maranhão, destaca-se a fitoterapia, realizada em 49,1\% dos municípios que ofertam Pics.

Plantas medicinais e fitoterápicos são amplamente utilizados em práticas por todo o Brasil, por serem facilmente acessíveis, pela abundância de diferentes espécies vegetais nativas no País, por apresentarem eficácia muito boa e baixo custo para algumas preparações, oferecendo alternativas de tratamento e supressão de carências medicamentosas ${ }^{16}$.

Entre as práticas instituídas pela PNPIC, a acupuntura e a auriculoterapia vêm apresentando tendência de crescimento em todo o País. Diversos municípios brasileiros estão realizando atendimentos e informando-os no SUS. No Maranhão, estas práticas estão sendo realizadas em $18,2 \%$ e $16,4 \%$ dos municípios que ofertam Pics, respectivamente ${ }^{17}$.

A publicação de Tesser, Sousa e Nascimento (2018) ${ }^{12}$ destaca, também, que um mesmo município ou estabelecimento de saúde pode ofertar mais de uma modalidade de Pics, o que vai de encontro ao observado no estado do Maranhão. Neste, $40 \%$ dos municípios que ofertam Pics disponibilizam mais de um tipo de prática. Corroborando com o presente estudo, os autores consideram que aproximadamente $78 \%$ desta oferta está na APS, sobretudo na ESF e nos Nasf.

Segundo os autores supracitados, na ESF, durante o ano de 2016, foram contabilizadas 2.427.919 ações em Pics, o que corresponde a 2.203.661 atividades individuais e 224.258 atividades coletivas ${ }^{12}$.

Os profissionais da ESF e do Nasf sem registro específico em Pics são considerados os protagonistas nesse campo. No Maranhão, destaca-se que os fisioterapeutas $(54,5 \%$ dos municípios que ofertam Pics) e enfermeiros (18,2\% dos municípios que ofertam Pics) são os profissionais que mais realizam tais práticas.

Pouco se sabe acerca dos profissionais que praticam as Pics no SUS, uma vez que os dados disponíveis são insuficientes, pois a maioria 
destas práticas pode ser realizada por profissionais da ESF sem registro específico ou vínculo formal em Pics. Segundo os registros no Cadastro Nacional dos Estabelecimentos de Saúde (CNES), a maioria dos profissionais que estão registrados realizando Pics no Brasil tem registro específico em Pics (4.104), com predominância de acupunturistas (médicos, $8,4 \%$; e fisioterapeutas, $19 \%$ ) e homeopatas (médicos, 31,8\%) ${ }^{\mathbf{1 2}}$.

A região de Codó é a que possui maior percentual de municípios realizando Pics, com $50 \%$ deles ofertando alguma prática. Destaca-se que Codó (MA) é conhecido não apenas como um município, mas como um espaço onde se produziu uma polissemia de representações, norteadas por interesses culturais, religiosos e sociopolíticos, a tal ponto de se tornar conhecido nacionalmente. Tais informações relacionam-se à maior oferta de práticas ${ }^{\mathbf{2 1}}$.

Na perspectiva de consolidação das Pics no SUS, destaca-se a necessidade de regulamentação das práticas através da criação de políticas municipais e de leis específicas'. Isto foi verificado no presente estudo, pois, segundo os gestores, nenhum município do estado apresenta lei específica para as Pics.

É responsabilidade da gestão municipal a elaboração de normas técnicas para a inserção da PNPIC na rede de saúde, a definição orçamentária e financeira para a implementação da política, a articulação intersetorial e a qualificação dos profissionais de saúde ${ }^{6}$.

Dessa forma, destaca-se que a institucionalização e oferta das Pics no SUS é um desafio para os gestores públicos. É necessário o envolvimento de atores sociais, instituições e profissionais, de forma participativa, na implantação das Pics na APS'.

Além disso, estudos relatam diversas dificuldades para a instituição das políticas públicas de Pics, como a deficiência de dados de pesquisa; os ciclos de mudança na gestão, que interrompem a continuidade dos processos de trabalho; a rotatividade dos profissionais que atuam na assistência; e a falta de direção, organização e controle das ações ${ }^{\mathbf{1 8}}$.
Esses desafios para a implementação das Pics nos sistemas de saúde não ocorrem somente no Brasil. Destaca-se que uma pesquisa realizada em 39 países europeus observou que $70 \%$ deles não possuem regulamentação específica sobre as Pics, ressaltando que somente a regulamentação não garante sua expansão sustentável na APS, sendo fundamental que a implantação das práticas terapêuticas nos serviços ocorra de maneira progressiva, descentralizada, atendendo às especificidades locorregionais, para o favorecimento dos processos de desenvolvimento das ações ${ }^{19}$.

Isso demonstra a potencialidade do presente estudo na divulgação de dados que tragam um diagnóstico das Pics no estado, diante da inexistência de uma política estadual para tais práticas, ainda que seja possível ressaltar algumas limitações que podem estar relacionadas à coleta de dados, principalmente pelo fato de estarem ligadas totalmente ao conhecimento do gestor de saúde local, que muitas vezes tinha pouco tempo no cargo.

\section{Considerações finais}

A implantação da PNPIC na APS se apresenta como operacionalmente simples, porém, ainda configura um desafio para as gestões estadual e municipal, uma vez que não há recurso específico para a política, o número de profissionais capacitados para a realização das Pics ainda é escasso, e a divulgação e o conhecimento sobre a política são incipientes. Acrescentese, ainda, a inexistência de uma coordenação nacional oficial da PNPIC no âmbito do MS, bem como a ausência de legislação estadual acerca das Pics.

O presente estudo identificou a realização de Pics no Maranhão. Dos municípios do estado, 25,4\% realizam algum tipo de Pics, e as práticas mais identificadas foram fitoterapia e massoterapia, estando presentes em $49,1 \%$ e 29,1\% dos municípios, respectivamente.

A ESF tem sido protagonista na execução das Pics na APS, seguindo as orientações da 
PNPIC, que destaca a APS como prioritária para as práticas, entendendo a contribuição destas para a resolutividade, o cuidado à saúde da população e a promoção da saúde.

Os achados do presente estudo destacam a importância de conhecer a situação do estado com relação às Pics, para que se possa pautar a construção de políticas públicas para o setor, uma vez que as políticas estabelecem diretrizes para as ações de implementação; os mecanismos de financiamento para as atividades; a elaboração de normas técnicas e operacionais; e a articulação com as demais políticas do MS.

Para a consolidação das Pics na APS é necessária a regulamentação das práticas, de forma a garantir a legitimação profissional e institucional, uma vez que é responsabilidade da gestão a elaboração de normas técnicas para a inserção da PNPIC na rede de saúde, bem como a definição orçamentária e financeira para a implementação da política, a articulação intersetorial e a qualificação dos profissionais de saúde.

Faz-se necessário o investimento, pela gestão estadual de saúde, na construção de uma Política Estadual de Práticas Integrativas e Complementares, de forma a instituir estas práticas no estado, bem como investimentos na divulgação e no conhecimento das Pics, para os gestores municipais de saúde. É necessário integrar gestores, profissionais de saúde e sociedade para promover discussões sobre a importância das Pics, sua utilização, benefícios e sua implementação no SUS.

A qualificação dos trabalhadores do SUS em relação a essas práticas também é necessária para garantir o protagonismo e empoderamento destes profissionais em relação à utilização das Pics em suas práticas assistenciais.

A incorporação das Pics na formação acadêmica também é uma ação que tende a fortalecer e ampliar a oferta das práticas, uma vez que ainda há escassez de profissionais qualificados.

Dessa forma, essas ações impactarão no aumento da oferta de Pics no estado e em sua consolidação no cuidado à saúde, promovendo, assim, a autonomia dos pacientes e a redução dos custos do SUS.

\section{Colaboradoras}

Soares RD (0000-0001-9896-5318)* contribuiu substancialmente para a concepção e o planejamento, para a análise e a interpretação dos dados. Pinho JRO (0000-00018857-8138)* contribuiu como orientadora de toda a construção do artigo. Tonello AS (0000-0002-8402-9112)* contribuiu para a construção dos resultados e como revisora do artigo final. 


\section{Referências}

1. Sousa IMC, Bodstein RCA, Tesser CD, et al. Práticas integrativas e complementares: oferta e produção de atendimentos no SUS e em municípios selecionados. Cad. Saúde Pública. 2012; 28(11):2143-54.

2. Sousa LA, Barros NF. Integrative and Complementary Practices in the Unified Health System: progresses and challenges. Rev Latino-Am Enf. 2018; 18(1). [acesso em 2020 ago 14]. Disponível em: http://www. scielo.br/scielo.php?script=sci_arttext\&pid=S0104$-11692018000100700 \& \operatorname{lng}=$ en.

3. Zanella AK, Ramires CC, Rocco CP, et al. Proposta de intervenção ensino-serviço de Práticas Integrativas e Complementares. VITTALLE. 2018; 30(1):63-71.

4. Gustavo LC, Correa AAM, Gualtierre A, et al. Conhecimento, acessibilidade e aceitação das práticas integrativas e complementares por usuários do sistema único de saúde em Juiz de Fora-MG. Rev Cient Fagoc. 2018; (3)9-15.

5. Política Nacional de Práticas Integrativas e Complementares no SUS: uma ação de inclusão. Ciênc. Saúde Colet. 2017 [acesso em 2020 ago 14]; 11(3):850. Disponível em: http://www.scielo.br/scielo.php?script=sci arttext\&pid=S1413-81232006000300034\&lng=en.

6. Brasil. Ministério da Saúde. Departamento de Atenção Básica. Política Nacional de Práticas Integrativas e Complementares no SUS: atitude de ampliação e acesso. 2. ed. Brasília, DF: Ministério da Saúde, 2015.

7. Azevedo C, Moura CC, Corrêa HP, et al. Práticas integrativas e complementares no âmbito da enfermagem: aspectos legais e panorama acadêmico-assistencial. Esc. Anna Nery Rev. Enferm. 2019; 23(2):1-9.

8. Telesi Júnior E. Práticas integrativas e complementares em saúde, uma nova eficácia para o SUS. Estudos Avançados. 2016; 30(86):99-112.

9. Mazon LM, Colussi CF, Senff CO, et al. Execução financeira dos blocos de financiamento da saúde nos municípios de Santa Catarina, Brasil. Saúde debate. 2018; 42(116):38-51.

10. Christina L, Barros N, Synthia E, et al. Os Sentidos das Práticas Integrativas e Complementares na Atenção Primária em Saúde para Gestores dos Serviços. Atas CIAIQ2018 Investigação Qualitativa em Saude. 2018; 2:1049-57.

11. Gontijo MBA, Nunes MF. Práticas Integrativas e Complementares: Conhecimento e Credibilidade de Profissionais do Serviço Público de Saúde. Trab. Educ. Saúde. 2017; 15(1):301-20.

12. Tesser CD, Sousa IMC, Nascimento MC. Práticas Integrativas e Complementares na Atenção Primária à Saúde brasileira. Saúde debate. 2018; 42(esp1):174-88.

13. Santos VR, Santos KOB. Fisioterapia e Práticas Integrativas e Complementares nos Núcleos de Apoio à Saúde da Família. Rev. Pesq. Fisiot. 2017; 7(2):207.

14. Galavote HS, Zandonade E, Garcia ACP, et al. O trabalho do enfermeiro na Atenção Primária à Saúde. Esc. Anna Nery Rev. Enferm. 2016; 20(1):90-8.

15. Camacho TSDA. Gestão: um Desafio Para o Enfermeiro. In: anais do $9^{\circ}$ Congresso Nacional de Excelência em Gestão; 2015 ago 13-14. Niterói: Universidade Federal Fluminense; 2015. [acesso em 2020 ago 4]. Disponível em: https://www.inovarse.org/sites/ default/files/T_15_067M.pdf.

16. Becker IRT, Cândido MR. Diagnóstico situacional de PIC. 2016. [acesso em 2020 ago 4]. Disponível em: http://repositorio.unesc.net/bitstream/1/719/1/ Mar\%C3\%ADlia\%20Raupp\%20C\%C3\%A2ndido.pdf.

17. Nunes MF, Junges JR, Gonçalves TR, et al. Práticas integrativas e complementares no âmbito da enfermagem: aspectos legais e panorama acadêmico assistencial. Saúde e Sociedade. 2017; 26(1):300-11.

18. Losso LN, Freitas SFT. Avaliação do grau da implan- 
tação das práticas integrativas e complementares na Atenção Básica em Santa Catarina, Brasil. Saúde debate. 2017; 41(esp3):171-87.

19. Wiesener S, Salamonsen A, Fonnebo V. Which risk understandings can be derived from the current disharmonized regulation of complementary and alternative medicine in Europe? BMC Complement Altern Med. 2018 [acesso em 2020 ago 4]; 18(1). Disponível em: https://bmccomplementmedtherapies.biomedcentral.com/articles/10.1186/s12906-017-2073-9.

20. Azevedo E, Pelicioni MCF. Práticas integrativas e complementares de desafios para a educação. Trabalho, Educação e Saúde. 2011; 9(3):361-78.

21. Oliveira RC, Carvalho e Silva MD. A cidade e os terreiros: os zeladores de santo e a construção do imaginário sobre Codó-MA. Mosaico. 2018 [acesso em 2019 nov 22]; 9(14):379-393. Disponível em: http:// bibliotecadigital.fgv.br/ojs/index.php/mosaico/article/view/73820/73235.

22. Mendes DS, Moraes FS, Lima GO, et al. Benefícios das práticas integrativas e complementares no cuidado de enfermagem. Journal Health NPEPS. 2019 jan-jun; 4(1):302-318.

23. Paiva LF. Práticas integrativas e complementares exercidas pelos profissionais de saúde no SUS: uma revisão sistemática sem metanálise. [dissertação]. Florianópolis: Universidade Federal de Santa Catarina, 2016. $177 \mathrm{p}$.
24. Costa ASA, Costa ASV, Cordeiro EC, et al. Entre o local e o nacional: os desafios contemporâneos na gestão da política nacional de práticas integrativas e complementares em saúde direcionados para a atenção básica, no município de São Luís-MA. Braz. J. of Develop. 2020; 6(4):17072-17085.

25. Nascimento MVN, Oliveira IF. As práticas integrativas e complementares grupais e sua inserção nos serviços de saúde da atenção básica. Estud. psicol. 2016; 21(3):272-281.

26. Souza EFAA, Luz MT. Bases socioculturais das práticas terapêuticas alternativas. Hist. cienc. saude-Manguinhos. 2009; 16(2):393-405.

27. Andrade JT, Costa LFA. Medicina complementar no SUS: práticas integrativas sob a luz da Antropologia médica. Saude soc. 2010; 19(3):497-508.

28. Instituto Brasileiro de Geografia e Estatística. Cidades e Estados [acesso em 2019 ago 27]. Disponível em: http:www.ibge.gov.br.

29. Maranhão. Secretaria de Estado da Saúde do Maranhão. Resolução no 64/2018 - CIB/MA. SES-MA, São Luís, 2018. [acesso em 2020 ago 4]. Disponível em: https://www.mpma.mp.br/arquivos/COCOM/ Resolu\%C3\%A7\%C3\%A3o_CIBMA_n\%C2\%BA_64_ de_25_de_maio_de_2018.pdf.

Recebido em 04/12/2019

Aprovado em 08/06/2020

Conflito de interesses: inexistente

Suporte financeiro: não houve 